\title{
Mineral Fabrication and Golgi Apparatus Activity in the Mouse Calvarium
}

\author{
Valerie Fallon', D. Howard Carter ${ }^{2}$, Jean E. Aaron'1 \\ ${ }^{1}$ Institute of Membrane and Systems Biology, Faculty of Biological Sciences, University of Leeds, Leeds, UK \\ ${ }^{2}$ Turner Dental School, University of Manchester, Manchester, UK \\ Email: j.e.aaron@leeds.ac.uk
}

Received 20 May 2014; revised 4 July 2014; accepted 16 July 2014

Copyright (C) 2014 by authors and Scientific Research Publishing Inc.

This work is licensed under the Creative Commons Attribution International License (CC BY). http://creativecommons.org/licenses/by/4.0/

(c) $)$ i) 0 pen Access

\begin{abstract}
There is diverse opinion about the mechanism of bone mineralization with only intermittent reports of any direct organellar role played by the golgi apparatus (juxtanuclear body). Light and laser confocal microscopy was combined with electron microscopy and elemental EDX (energy dispersive X-ray microanalysis) in comparing "young" osteocytes in situ in fresh and "slam" frozen developing mouse calvarium, with similar cells (MC3T3-E1) maintained in vitro. The distribution of "nascent" electron dense mineral was examined histochemically (von Kossa, GBHA), including tetracycline (TC) staining as a fluorescent complex with bone salt, while golgi body activity was demonstrated by transfection with a specific green fluorescent construct (GFP/mannosidase II). In tissue culture golgi body activity and mineralization were both blocked by brefeldin A (an established golgi inhibitor) and restored by forskolin, enabling an association with mineral fabrication to be quantified as changing fluorescence intensity (AU) of GFP or TC markers. Results from osteocytes in situ supported previous descriptions of intracellular electron dense objects (microspheres and nanospheres) in a juxtanuclear pattern, containing $\mathrm{Ca}, \mathrm{P}$ and transitory $\mathrm{Si}$, in a spectrum recapitulated in the calcifying culture after 10 days, when GFP fluorophore surged from 71.7 $\pm 1.4 \mathrm{SD}$ to $133.7 \pm 2.7 \mathrm{SD}$ AU by 14 days $(p<0.0001)$. At this stage TC fluorophore mean intensity was $23.8 \pm 3.7 \mathrm{SD}$ AU (14 days) rising to 45.0 \pm 5.1SD AU by 17 days, compared to its stationary 21.7 $\pm 3.6 S D$ when treated 3 days previously with BFA golgi inhibitor $(p<0.0001)$, until forskolin reversal. It was concluded from the changing juxtanuclear morphology, Si mineralization mediation and the variably controlled activity versus stasis that the inorganic phase of bone is a complex golgi-directed fabrication with implications for bone matrix biology and evolution.
\end{abstract}

\section{Keywords}

Bone Biomineraliization, Golgi Apparatus, Tetracycline Fluorochrome, GFP Fluorochrome, Brefeldin A/Forskolin 


\section{Introduction}

In examining events at the calcification front of bone it has been long recognized that the first-formed (i.e. nascent) bone mineral is soluble, readily transformed by exposure to chemicals and is easily lost during processing, resulting in a diversity of views in the literature about whether the mineralization process commences extracellularly or intracellularly. High velocity impact (slam) freezing is an instantaneous cryo-preparative technique considered to conserve tissue chemistry optimally in small bone specimens which risk losing some of their nascent bone mineral with more prolonged processing [1] [2]. The developing calvarium of the young mouse is a particularly useful model for examining osteocytes in situ because it is optically thin enabling 3-dimensional imaging without disturbance and requiring minimal preparation which is ideal for slam freezing [3]. At the same time, there is a bone forming cell line MC3T3E1 derived from newborn mouse calvaria [4] that displays histochemical and biochemical characteristics of bone synthesis and develops a network of dendritic processes that culminate in the calcification of an extracellular matrix in vitro when supplemented with organic phosphate [5] (see also [6]).

It has been previously reported histochemically [7] [8] that there may be a relationship between juxtanuclear body (golgi apparatus viewed optically) activity, with expansion of its reticular area, and calcium phosphate production. This is especially evident when tetracycline (TC), which has a long history in association with bone mineralisation as a tissue time marker, is used as a mineral stain with which it forms a fluorescent complex [9]. Intracellularly this is coincident with the juxtanuclear body. Evidence concerning TC as a marker of mineralisation [10] [11] has indicated that the binding is not a simple chelation of TC with calcium but is a stable complex with bone salt. Its apparent affinity for the calcification front and adjacent cell juxtanuclear bodies was therefore of special interest. More recently there has been identified within the golgi membrane stack a permanent resident protein, visualised as a green fluorescent protein (GFP)/mannosidase II construct in validated transfection assays and now used widely in biology as a reliable marker of golgi activity [12].

It is proposed to expand upon the previous static bone histology which used the von Kossa stain for phosphate/carbonate and Kashiwa's GBHA for calcium [13], to now include TC fluorochrome for bone salt, together with elemental (EDX; energy dispersive X-ray) microanalysis applied quantitatively to bone cell culture dynamics. The aim is firstly, to confirm the optical status of the juxtanuclear body, bone mineral and related X-ray microanalysis in situ in "young" osteocytes of the developing mouse calvarium; secondly, it is to determine the corresponding laser confocal status in vitro using GFP construct and TC fluorochrome as monitors of organellar mineral fabrication. To this end the established biological golgi-inhibitor Brefeldin A (BFA) is used to "switch off" the juxtanuclear body to determine any coincident effect on cell-directed mineralisation, while the agent forskolin is used to restore, i.e. "switch on" activity [14].

\section{Methods}

\section{1. "Slam" Freezing/EDX Mineral Microanalysis, Structure and Substructure in Situ}

Transverse, unstained, ultrathin sections of slam-frozen neonate mouse calvarium ( 1 - 5 days old) were prepared as previously reported in detail [3] by impact of the tissue with a copper block cooled by liquid nitrogen using MM80 freezing apparatus (Leica UK Ltd., Milton Keynes; courtesy of Prof. P. V. Hatton, University of Sheffield) followed by infiltration with Lowicryl K4M with polymerization by ultraviolet irradiation. Sections, 0.25 $\mu \mathrm{m}$ thick (i.e. unusually thick) were examined in a Philips CM10 electron microscope and EDX microanalysis of electron dense objects performed at the immature calcification front and in the more mature bone regions. Intact young mouse calvarium was also histochemically stained with von Kossa's silver stain (for phosphate/carbonate) and Kashiwa's GBHA stain (for calcium) as previously reported [15] [16] for optical comparison of the substructural detail.

\subsection{Laser Confocal GFP Golgi Marker of Activity in Vitro}

MC3T3-E1 cells (from neonate murine calvarium; a gift from Dr. P. Genever, University of York) were seeded (density $5 \times 10^{4} / \mathrm{mL}$ ) onto acetate coverslips, placed in a welled plastic plate and grown for 6 - 7 days in $\alpha$-MEM (minimum essential medium) containing 10\% FCS (foetal calf serum), penicillin (100 units/mL) and streptomycin $(100 \mu \mathrm{g} / \mathrm{mL})$, together with supplement ( $5 \mathrm{mM} \beta$-glycerophosphate and $25 \mu \mathrm{g} / \mathrm{mL}$ ascorbic acid phosphate [5] [6] in a humidified atmosphere $\left(5 \% \mathrm{CO}_{2}\right.$ and $95 \%$ air at $\left.37^{\circ} \mathrm{C}\right)$. Cells were transfected for a final 24 hours 
with (GFP)/mannosidase II construct (a gift of Dr. K. Zaal supplied by Dr. M. Peckham, University of Leeds) using FuGENE $6^{\mathrm{TM}}$ (Roche) transfection reagent, followed by fixation in $2 \%$ paraformaldehyde, $\mathrm{pH}$ 7. Control cells, not transfected were rinsed in deionized water and stained with the nuclear fluorophore TOPRO-3. After mounting in Antifade reagent (Molecular Probes, UK) they were examined under a Zeiss LSM 510 META laser scanning confocal microscope (GFP, excitation laser $488 \mathrm{~nm}$, emission filters to collect $509 \mathrm{~nm}$ wavelength; TOPRO-3, excitation laser $633 \mathrm{~nm}$, emission filters for $661 \mathrm{~nm})$. GFP fluorescence quantification was performed using the Imaris 4.1 (Bitplane) programme.

\subsection{Laser Confocal TC Marker of Mineral-Related Activity in Vitro}

MC3T3-E1 cells were seeded as above, fixed, rinsed and stained in tetracycline hydrochloride $(10 \mu \mathrm{g} / \mathrm{mL})$ for $30 \mathrm{~min}$ and examined under the laser confocal microscope (excitation laser $360 \mathrm{~nm} \pm 40$, emission filters to collect $528 \mathrm{~nm} \pm 38$ ). TC fluorescence quantification was performed using the Imaris 4.1 (Bitplane) programme.

\subsection{Laser Confocal Brefeldin Golgi "Switch off" and Forskolin Reversal for Mineral Fabrication in Vitro}

MC3T3-E1 cells were cultured in flasks containing $\alpha$-MEM and 10\% foetal calf serum (FCS), with penicillin, streptomycin and calcifying supplement in a humidified atmosphere as above. At 3 days prior to analysis the cells were rinsed in calcium/magnesium-free phosphate buffered saline (PBS) and sub-cultured using 0.05\% trypsin to cause cell detachment. As a BFA toxicity assay, cells were seeded on acetate coverslips as above and grown for 14 days when BFA (10 nM) was added followed by cell growth for 3 - 4 more days before sampling for confocal microscopy. [Experimentation at higher BFA doses (200, 100 and $50 \mathrm{nM})$ compared with untreated controls resulted in cell death at approximately 4,12 and $36 \mathrm{hr}$ respectively]. Subsequent supplementation with forskolin (100 $\mu \mathrm{M}$ for $30 \mathrm{~min}, 1,2$ and 3 hours respectively) in the continued presence of BFA followed. Also included was a test for apoptosis by adding propidium iodide $(1 \mu \mathrm{l} / 5 \mathrm{~mL})$ to cultures for the remaining 20 min of the investigation (excitation laser $536 \mathrm{~nm}$, emission filters for $620 \mathrm{~nm}$ ). TC and GFP fluorescence quantification was performed throughout using the Imaris 4.1 (Bitplane) programme above. The statistical analysis of the data as two sample t-tests and ANOVA was performed using Minitab Version 14 and Genesis software.

\section{Results}

\section{1. "Slam" Freezing/EDX Mineral Microanalysis, Structure and Substructure in Situ}

Electron dense calcified objects (Figure 1) in the calvarial sections and also visible under the optical microscope in whole mounts ranged from 30 - $50 \mathrm{~nm}$ dense bodies to $5 \mathrm{~nm}$ nanoparticles assembled within filamentous clusters up to $1 \mu \mathrm{m}$ in diameter, found within the "young" osteocyte and outside the cell (Figure 2), as previously reported [3]. Elemental mapping of the spherical intracellular objects supported the presence of calcium and phosphorus, and also a transitory silicon peak which was not found in more mature regions of the matrix. It apparently related to the initiation and modulation of the bone salt inside the "young" osteocyte, demonstrated in the juxtanuclear region optically (Figure 1(a), showing the von Kossa stain for phosphate/carbonate in combination with the GBHA calcium stain of Kashiwa (1970) and ultrastructurally Figure 1(b)), in both sections and in whole mounts of the optically thin calvaria at this neonatal stage of development.

\subsection{Laser Confocal GFP Construct Golgi Marker of Activity in Vitro}

MC3T3-E1 cultured control cells had well-defined nuclei with many nucleoli as shown by TOPRO-3 stain and those transfected with GFP/mannosidase II construct showed fluorescence located in the juxtanuclear region (Figure 3). Inspection of supplemented cultures at intervals of 7 to 14 days showed a progressive time-dependent golgi fluorophore mean intensity to a maximum at 14 days (Figure 4), increasing from $34.6 \pm 1.4 \mathrm{SD}$ AU (arbitrary units) to $133.7 \pm 2.7 \mathrm{SD}$ AU (Table 1 ; differences between groups examined for significance by analysis of variance ANOVA $\mathrm{p}<0.0001)$.

\subsection{Laser Confocal TC Marker of Mineral-Related Activity in Vitro}

Cell-free control incubation of the supplemented medium showed no sign of mineral deposition, neither did cell 

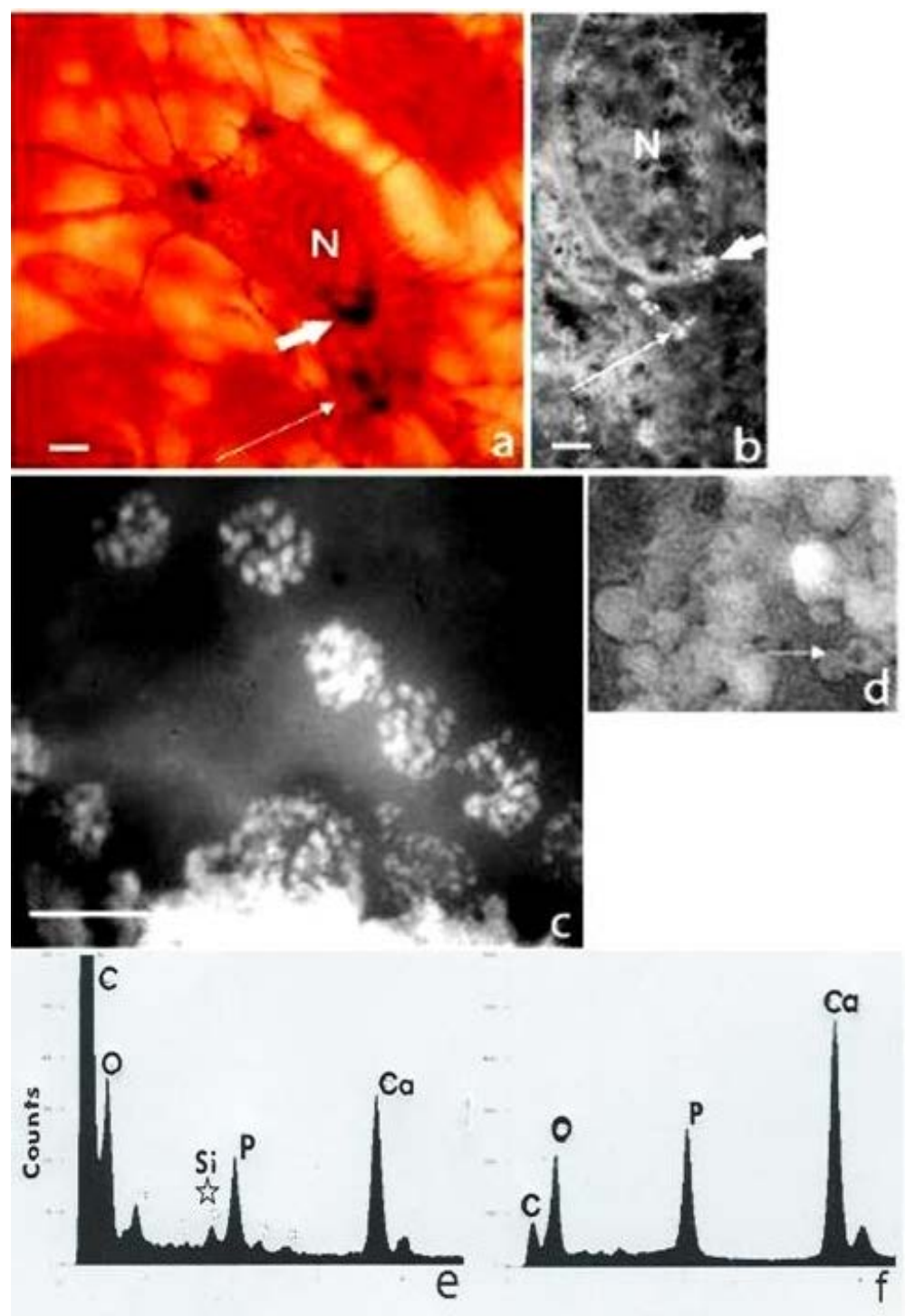

Figure 1. Bone cells and mineral particles in situ in the 5-day-old mouse calvarium. (a) Intact osteocyte stained histochemically for calcium (GBHA stain) and phosphate/carbonate (von Kossa stain) showing the nucleus (N) and a juxtanuclear crescent (large arrow) composed of dark microspheres $(<1$ $\mu \mathrm{m})$, together with peripheral groups (small arrow); H. P. incident light interference contrast (Nomarski) optics scale bar $4 \mu \mathrm{m}$. (b) Similar osteocyte in section, "slam" frozen and unstained showing a juxtanuclear crescent and peripheral groups of white microspheres (arrowed), each composed of dense bodies $(30-50 \mathrm{~nm}$ ); L. P. TEM (unstained, negative image, i.e. electron dense mineral white for maximum resolution), scale bar $3 \mu \mathrm{m}$. (c) Juxtanuclear microspheres $(1 \mu \mathrm{m}$ in diameter) in detail as clusters of nanospheres (30 - $50 \mathrm{~nm}$ in diameter), with (e) a corresponding EDX spectrum showing Ca and P peaks together with Si (below star); H. P. TEM, scale bar $1 \mu \mathrm{m}$. (d) Electron dense microsphere populations $(1 \mu \mathrm{m}$ diameter) in the calcified extracellular matrix (some with less dense centres, arrowed) and (f) with a corresponding EDX spectrum showing $\mathrm{Ca}$ and $\mathrm{P}$ peaks and no $\mathrm{Si}$

cultures without the $\beta$-glycerophosphate/ascorbic phosphate supplement. MCT3TC-E1 cultured control cells showed only a dull autofluorescence at the outset i.e. they were apparently not fabricating fluorescent mineral particles for several days, as monitored by von Kossa and alizarin red (calcium) stains. Inspection of supple 

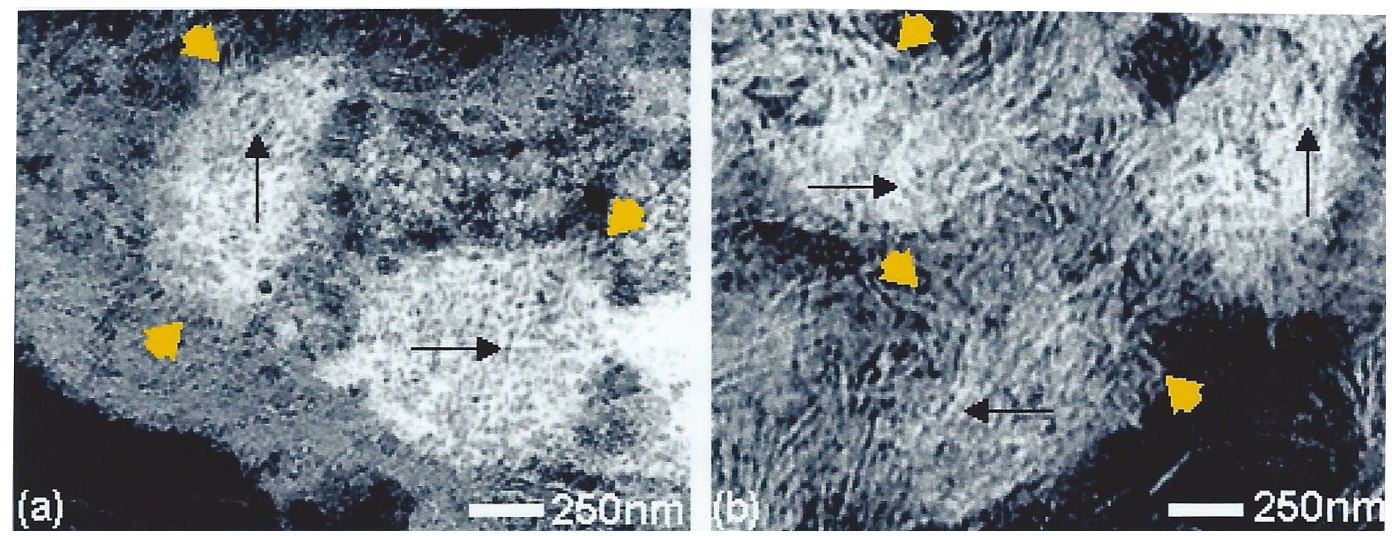

Figure 2. Substructure of intracellular (a) and extracellular (b) mineral particles in situ in undecalcified bone. Showing micron-sized electron dense objects as clusters (yellow arrowheads; $1 \mu \mathrm{m}$ in diameter) of beaded (black arrows) filaments ( $5 \mathrm{~nm}$ wide). TEM, stained for contrast (negative image i.e. mineral white).
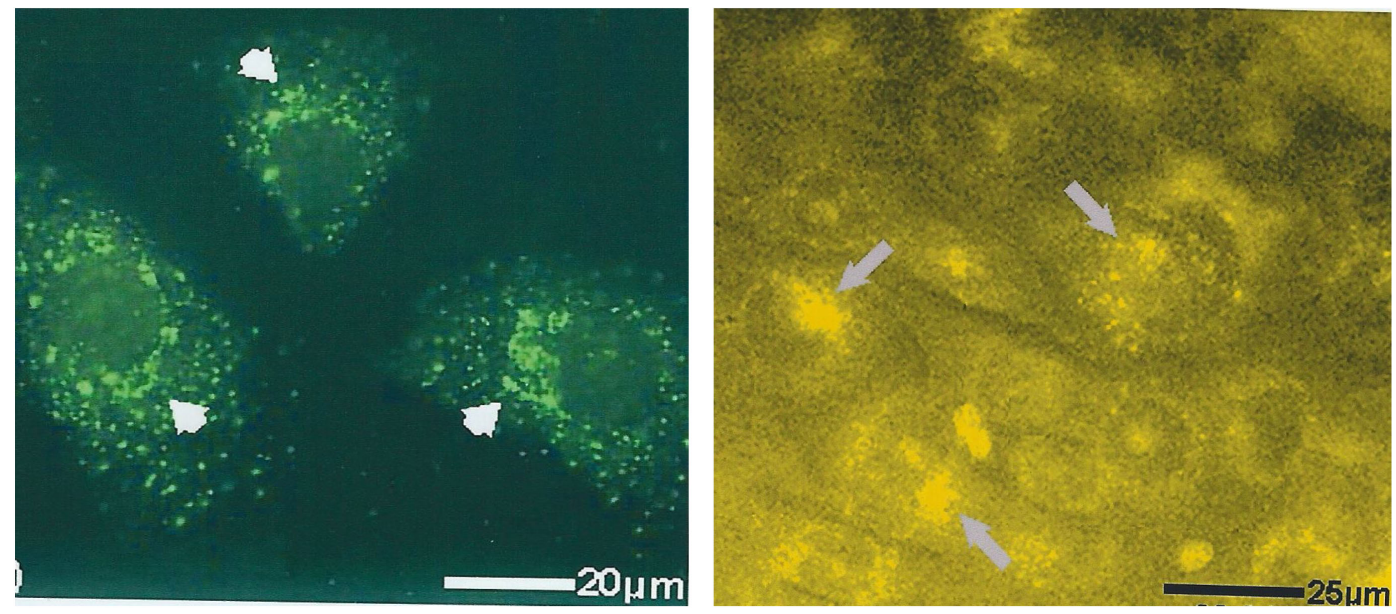

Figure 3. Fluorescent markers of the golgi apparatus (arrowed) in individual bone cells (MC3T3-E1) in vitro. Showing examples of the crescent-shaped juxtanuclear fluorescence (left) with GFP/mannosidase II construct and (right) with TC stain. Laser confocal microscope.

Table 1. Cultured bone cell (MC3T3-E) mean fluorescence intensity of GFP mannosidase II construct for golgi complex activity and of TC (tetracycline) for bone salt fabrication in 6 assays $(\mathrm{n}=6)$ after 7, 9 and 14 days showing the time-related fluorochrome surge in both. A specific TC filter removes the autofluorescence component (13.5 AU) and columns in brackets were below the 13.5 threshold until after 9 days when previous engagement in collagen precursor synthesis switches to mineral fabrication. Laser confocal microscopy (AU arbitrary units). (ANOVA p < 0.0001).

\begin{tabular}{ccccccc}
\hline Culture & \multicolumn{7}{c}{ Fluorescence Intensity (AU) } & \multicolumn{2}{c}{ TC } \\
\hline Assay & \multicolumn{7}{c}{ GFP } & & $\mathbf{1 4}$ days & $\mathbf{7}$ days & $\mathbf{9}$ days & $\mathbf{1 4}$ days \\
\hline 1 & $\mathbf{7}$ days & $\mathbf{9}$ days & 132.2 & $(11.3)$ & $(12.6)$ & 21.5 \\
2 & 32.7 & 69.3 & 131.0 & $(9.9)$ & $(13.1)$ & 28.6 \\
3 & 36.1 & 72.7 & 133.4 & $(10.1)$ & $(12.9)$ & 32.5 \\
4 & 34.7 & 71.9 & 137.6 & $(8.8)$ & $(10.8)$ & 20.3 \\
5 & 33.1 & 72.8 & 136.3 & $(8.3)$ & $(11.9)$ & 18.1 \\
6 & 35.2 & 70.4 & 131.8 & $(7.6)$ & $(11.5)$ & 31.6 \\
Mean & 35.9 & 72.6 & 133.7 & 0.0 & 0.0 & 25.4 \\
SD \pm & 34.6 & 71.7 & 2.7 & 0.0 & 0.0 & 6.2 \\
\hline
\end{tabular}



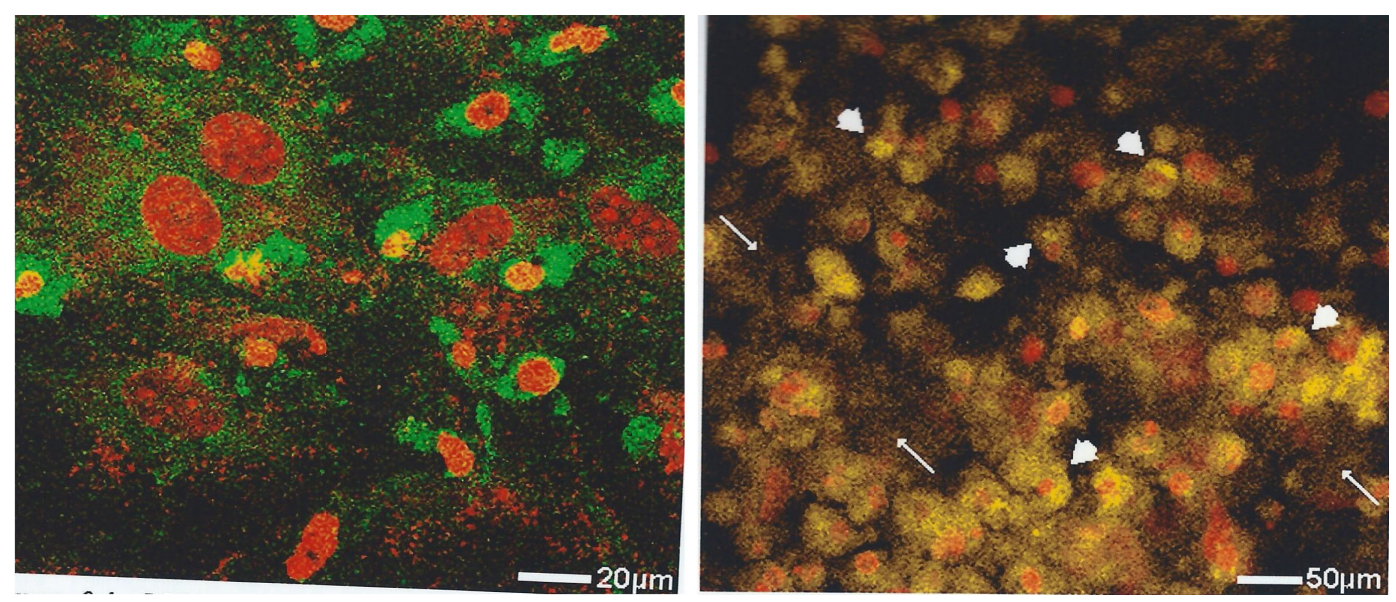

Figure 4. Fluorescent markers of golgi apparatus activity in bone cells (MC3T3-E1) in vitro after 14 days culture when their shape is typically irregular. Showing (left) the prominent intensity of the condensed juxtanuclear golgi apparatus and also its more extensive dispersion throughout the cytoplasm, GFP/mannosidase II construct; (right) the similar intracellular golgi-related distribution of the TC-tagged mineral (arrowheads), with some extracellular labelling (arrowed). Topro-3 nuclear stain (red). Laser confocal microscope.

mented cultures at intervals of 7 to 10 days showed an initial shape transformation from fusiform to cuboidal had taken place with continued negativity for TC-mineral binding. It was not until 10 days had elapsed when cell elongation to ovoid and stellate was recorded (Figure 3) and by 14 days was coincident with a mean fluorescence intensity of $25.4 \pm 6.2 \mathrm{SD}$ AU (Table 1). This derived from localised fluorescence observed in the juxtanuclear region (Figure 4) and with a paler extracellular fluorescence detected above the background autofluorescence, which suggested a sequence of events that was also followed by time-lapse microscopy as the passage of individual particles from cell interior to the exterior (Figure 5) and with an EDX spectrum characteristic of the calcification front in situ, including not only Ca and P but also Si (Figure 6).

\subsection{Laser Confocal Brefeldin and Forskolin Golgi "Switches" for Mineral Fabrication in Vitro}

MCT3TC-E1 cultured control cells exposed to BFA at 14 days was followed by the retraction of cytoplasmic processes during the subsequent 3 days, while the nuclei and nucleoli remained well defined and the propidium iodide stain for apoptosis was negative. However, more prolonged exposure beyond a further 4 days was associated with the appearance of features consistent with programmed cell death i.e. pyknotic nuclei and positive staining with propidium bromide for apoptosis. Observations were therefore confined to the 3 day window i.e. inspection of supplemented cultures at 17 days. At this stage, untreated cells stained intracellularly for mineral with von Kossa stain, with some extracellular stained particles bordering the cells which showed an increase in cell processes and a general confluence. At the same time histochemically stained juxtanuclear bodies were a prominent feature. In contrast, cells exposed continuously to brefeldin after 14 days optimal culture showed retraction of their processes, shrinkage and fragmentation of the juxtanuclear body and fewer mineral particles equivalent only to those present at 14 days, suggesting the arrest of mineral fabrication coincident with the BFA golgi "switch off."

To enable quantification of these subjective observations TC stain was applied to untreated and BFA-treated cells cultured optimally to 14 days and subsequently exposed to BFA for 3 days as above and the mean fluorescence intensity of the TC-mineral complex measured (Figure 7). A juxtanuclear concentration of fluorescence was supported with a time-dependent increasing intensity to 14 days, which stopped almost immediately in the presence of BFA ( $21.7 \pm 3.6 \mathrm{SD} \mathrm{AU})$ compared with untreated controls at $23.8 \pm 3.7 \mathrm{SD}$ AU which went on to reach $45.0 \pm 5.1 \mathrm{SD}$ AU, examined for significance $(n=6)$ by the two sample t-test $\mathrm{p}<0.0001$ (Figure 8 ). The BFA-treated cells were negative for apoptosis with propidium iodide stain indicating cell dormancy and not death. Verification of this with forskolin was indicated when 14 day cultures were treated with BFA for 3 days followed by the golgi "switch on" reversal agent. Within 3 hours there was an extension of the retracted cell processes, juxtanuclear body re-expansion from a shrunken state and a return to normal functioning. (See [17] 


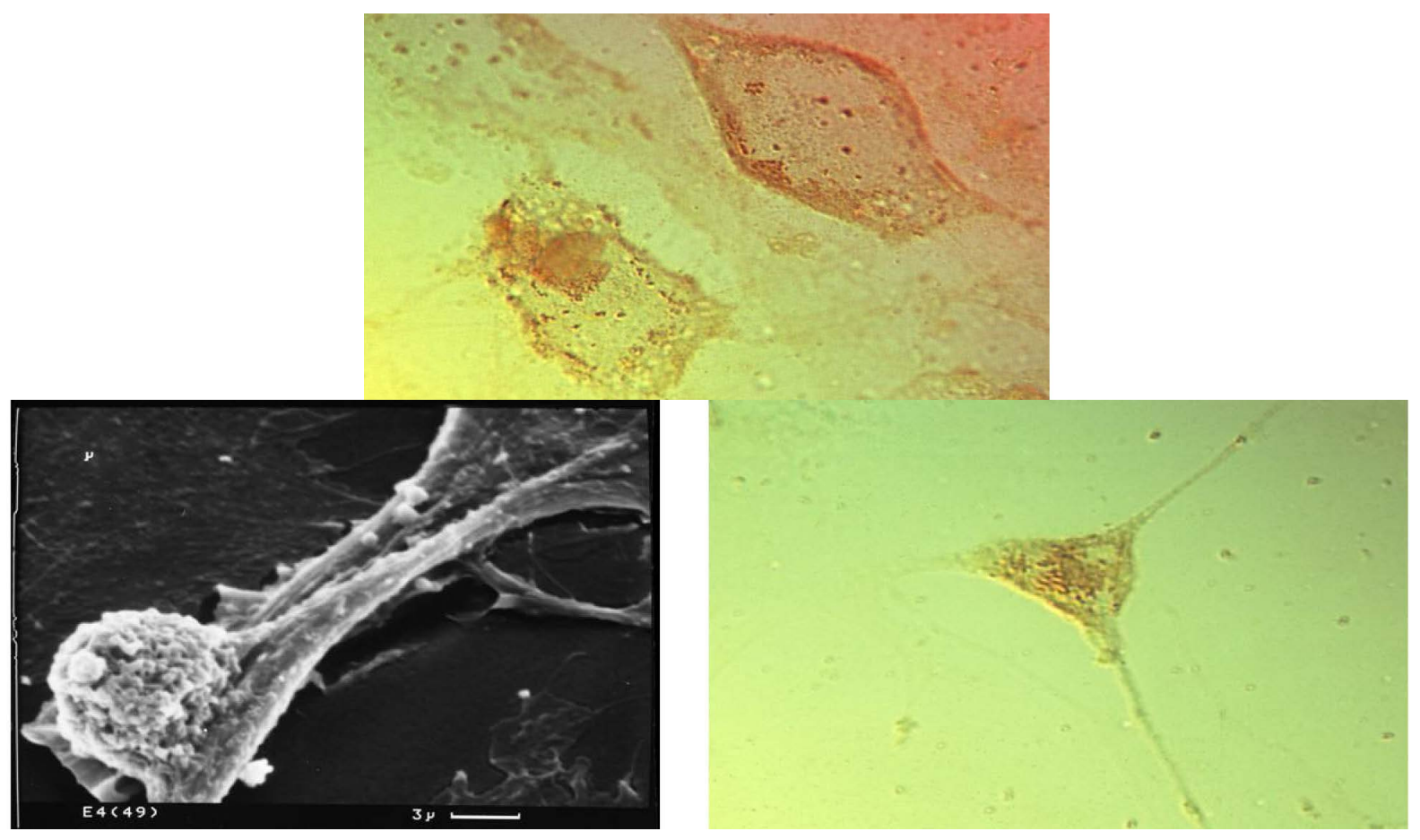

Figure 5. Bone cell (MC3T3-E1) mineral particle trafficking in vitro. Showing (top) a typical irregular cell with $1 \mu$ m intracellular mineral particles and (bottom right) elongated, slender cytoplasmic processes with mineral particles inside and outside (LM), and with one enlarged cell process (bottom left) bulging with a single calcified microsphere about $1 \mu \mathrm{m}$ in diameter (SEM).
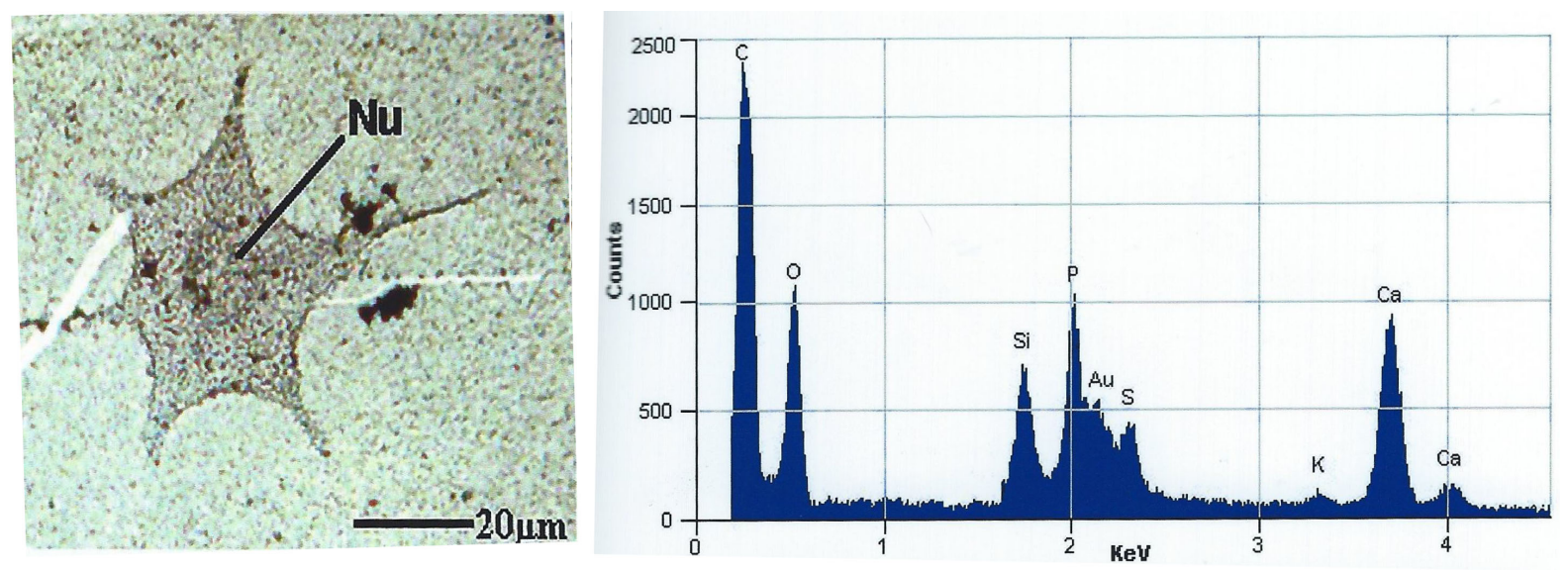

Figure 6. EDX microanalysis of bone mineral fabricated in vitro by 49 day cultured bone cells (MC3T3-E1). (Left) A typical intact stellate cell with a central nucleus $(\mathrm{Nu})$ and cytoplasmic mineral particles with extracellular assemblies and dispersed particles. Von Kossa stain. (Right) Typical elemental spectrum of cell isolates showing Ca and P peaks and also Si and S.

for further details and references).

\section{Discussion}

The absence of mineral particles from incubated cell-free, supplemented medium $(\beta$-glycerophosphate and ascorbic acid phosphate) suggests that the production observed was dependent upon the input of biological energy and not confined to physicochemical precipitation from a metastable solution. At the same time, the failure of cells grown without supplement either to fabricate calcified objects or to develop a related prominent golgi complex, implies that $\beta$-glycerophosphate/ascorbic phosphate supplement was essential for this particular phe- 


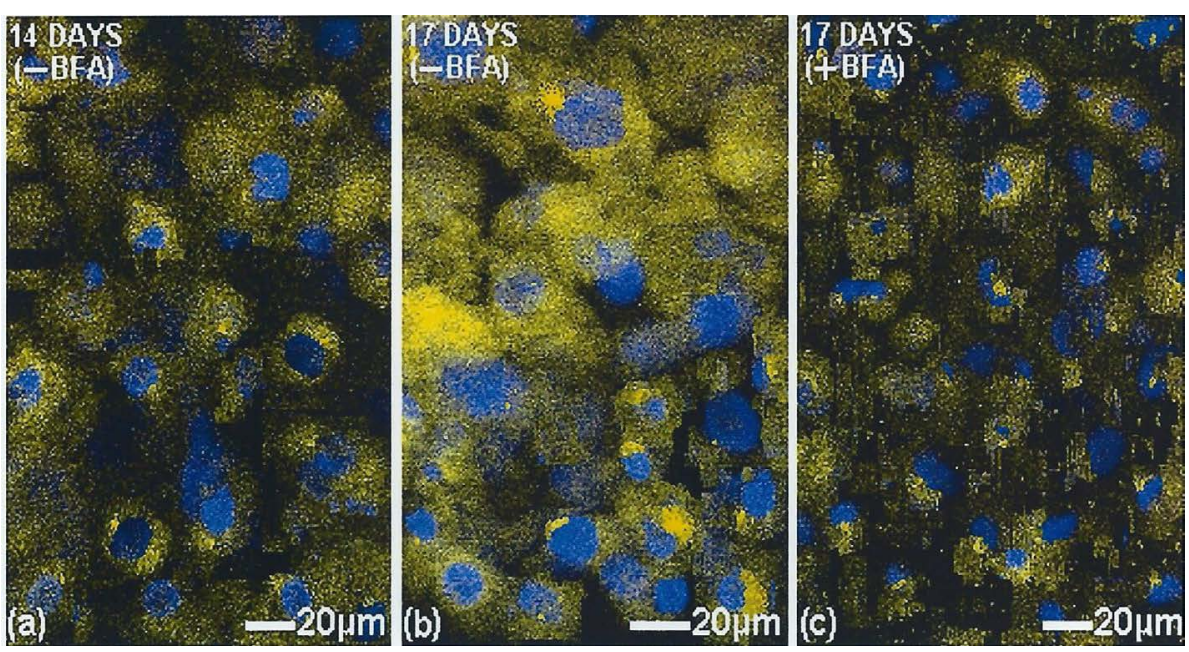

Figure 7. Golgi apparatus inhibition in vitro in 14 days cultured bone cells (MC3T3-E1) without and with added brefeldin A (BFA). Showing (a) the juxtanuclear TC-tagged mineral at 14 days and (b) its organellar expansion throughout the cytoplasm by 17 days, in contrast to (c) its organellar stasis with BFA. TOPRO-3 nuclear stain (blue). Laser confocal microscope.

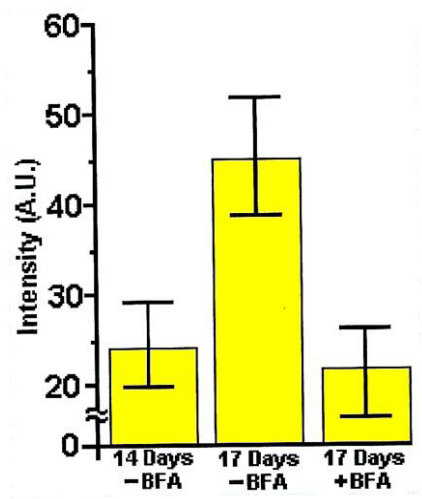

Figure 8. Histogram of tetracycline (TC)-tagged mineral inhibition in cultured bone cells (MC3T3-E1) by 17 days, with and without the golgi apparatus inhibitor brefeldin A (BFA) administered at 14 days. Mean fluorescence intensity with highest and lowest readings indicated (whiskers). Respective means $23.8 \pm 3.7 \mathrm{SD}, 45.0 \pm 5.1 \mathrm{SD}$ and $21.7 \pm 3.6 \mathrm{SD}$. (Each column represents 6 culture assays, $n=6$; t test $p<0.0001$ ).

notype expression. Just as the presence of silicon seems to be a key early intermediary of calcification with phosphate, featuring regularly from mineralizing bacteria [18] and protozoa [17] through to bone, so it is supported as a hallmark of "young" osteocytes at the calcification front in situ and in vitro above, indicating their mineral-related identity. In situ the populations of electron dense objects previously described [3] as calcified microspheres (about $1 \mu \mathrm{m}$ in diameter) and nanosphere precursors (30 - $50 \mathrm{~nm}$ ) are frequently to be seen assembled in a predictable juxtanuclear pattern evident optically and ultrastructurally (shown in Figure 1(b) without the benefit of the usual TEM membrane stains which risk transforming or eluting the earliest mineral deposits). There follows dispersal through the cytoplasm and processes (sometimes evident in terminal protruberances; see also [19]) to the exterior calcification front where they aggregate as filamentous (5 $\mathrm{nm}$ wide) clusters described in ultrastructural detail elsewhere [20]-[22]. As an established bone salt fluorochrome, TC has been used extensively for a tissue time marker because of its special affinity for the calcification front. At the same time it has been described as having an apparent similar affinity for the juxtanuclear body, which can vary in size from a small, compact and discrete ovoid feature to an expansive crescent with cytoplasmic reticulation depending upon the cell cycle of activity stimulated. The evidence suggests a relationship between golgi activity and calcified microsphere/nanosphere juxtanuclear production in "young" murine osteocytes in situ and cultured murine 
bone cells such that elevated mineral fabrication tagged with TC was synchronised with elevated golgi activity tagged with GFP and was modulated by BFA and forskolin. An optimal window for this mineral cell cycle is evident from about 10 days onwards, prior to which the golgi apparatus produces collagen precursor molecules, and post which it eventually enters a period of quiescence. In vivo this will last until stimulation by humoral or physical signals translated through the matrix.

By 35 days of optimal culture, nodular accretions about 10 microns in diameter resembled those described [5] as woven bone, positive for osteocalcin [23]. In situ there is a clear spatial separation between the superficial rows of cuboidal osteoblasts with well developed juxtanuclear bodies engaged in osteoid tissue formation for about ten days, and the "young" stellate osteocytes recently matrix-encapsulated with their golgi bodies progressively "switched off" for the organic components and "switched on" for the inorganic. However, in the sheets of contiguous cultured cells the modulation from one activity to another is less clear, even though at some stages the cells tend to be fusiform (early, i.e. fibroblast-like), at another they may be cuboidal (medium, i.e. osteoblast-like) and at another stellate (late; i.e. osteocyte-like). It may be no coincidence that although the bone cells are maintained in an in vitro state, their product before 9 days was apparently collagen alone, with mineral production engaged only after a period of 10 days had elapsed, i.e. these isolated bone cells may be responding to a stable inorganic biological clock as seems manifest in vivo. The cell cultures grown for 49 days with supplementation were the most calcified and the mineral phase could be separated by hydrazine hydrate organic digestion, when numerous micron-sized microspheres were isolated, singly and in chains. EDX confirmed the presence of calcium and phosphate (molar Ca:P ratio 1.01) and a pronounced Si peak [17]. Such mineral particles are not pure precipitates but have an integral organic component (for example, osteopontin, bone sialoprotein, osteocalcin; [24] [25]) that places the physical chemistry into compartments and envelopes, enabling biological control of natural physicochemical mineral growth [26].

Green fluorescent protein (GFP) is a natural jellyfish bioluminescent where it functions as an energy-transfer acceptor changing blue light emitting photoprotein aequorin into green and its use as a golgi marker in live cells is well established [27]. The description by Camillo Golgi in 1898 of an "internal reticular apparatus" in neurons based on apparently capricious heavy metal staining was given little consideration until its confirmation was enabled by the technological leap of electron microscopy and its role in intracellular trafficking and macromolecular assembly was established together with its close association with the rough endoplasmic reticulum. The drug brefeldin A blocks this exchange by specifically disrupting and dismantling the golgi apparatus and its secretory pathway with changes taking place within minutes. BFA is a fungal metabolite initially characterised as an antiviral antibiotic [28] and is now established as an essential tool in the lexicon of cell biology ([29] for review). Used in combination with forskolin the two provide the valuable mechanism of "on/off switches" to facilitate the examination of itinerant, golgi-derived structures.

In summary there is now available a bone biomineralization system that is apparently responsive to the BFA golgi apparatus "on/off" switch and three main conclusions can be drawn from the results. First, there are close similarities between the mouse cells in situ in the developing calvarium and those maintained in vitro in terms of intracellular and extracellular calcified particles, their elemental spectra including a significant Si peak [18] and their intermittent juxtanuclear affinity. Second, the histochemically identified juxtanuclear mineral, including TC fluorescence is morphologically, temporaly and spatially coincident with the fluorescence of a specific GFP golgi construct and enables intensity monitoring with time. Third, BFA-treatment known to induce golgi dormancy, is dynamically coincident with the reversible suspension of mineral fabrication, and seems more consistent with a direct golgi apparatus dependency than an indirect association. Thus, while physicochemical precipitation processes are a part of the bone mineral event, the regular assembly of calcified objects in the golgi body ensures their organic containment and their individual and independent inorganic character and size limitation for optimal function. Just as the modulating bone cell in vivo switches from osteoid synthesis after about ten days to mineral fabrication at the calcification front (as measured by tetracycline double labelling), so the golgi complex is reprogrammed and reorganized in vitro after about 9 days. Such golgi apparatus change is reminiscent of events established in skeletal muscle differentiation [30]. All this, combined with a propensity to respond in a directed rather than a random manner to external signals, is perhaps originally derived from the competitive advantage of calcification with phosphate acquired by the early protozoa for example, [31] [32] thereby founding a calcium phosphate pathway common to all mammalian cells to a greater or lesser degree. Any biomechanical and clinical implications arising from this biology may need to be defined in terms of the performance of the golgi apparatus. 


\section{Acknowledgements}

We are grateful to Dr. Roger C. Shore, Department of Oral Biology, University of Leeds, for guidance with EDX microanalysis, to Prof. Michelle Peckham for valuable advice about tissue culture procedures and together with Ruth Anderson-Beck for use of their facilities, and finally to Dr. F. G. E. Pautard for inspiration.

\section{References}

[1] Ross, G.D., Morrison, G.H., Sacher, R.F. and Staples, R.C. (1983) Freeze Substitution Sample Preparation for Ion Microscopy of Plant Tissue. Journal of Microscopy, 129, 221-228. http://dx.doi.org/10.1111/j.1365-2818.1983.tb04176.x

[2] Hunziker, E.B., Hermann, W., Schenk, R.K., Mueller, M. and Moor, H. (1984) Cartilage Ultrastructure after High Pressure Freezing, Freeze Substitution and Low Temperature Embedding. I. Chondrocyte Ultrastructure-Implications for the Theories of Mineralisation and Vascular Invasion. The Journal of Cell Biology, 98, 267-276. http://dx.doi.org/10.1083/jcb.98.1.267

[3] Carter, D.H., Hatton, P.V. and Aaron, J.E. (1997) The Ultrastructure of Slam-Frozen Bone Mineral. The Histochemical Journal, 29, 783-793. http://dx.doi.org/10.1023/A:1026425404169

[4] Kodama, H.-A., Amagai, Y., Sudo, H., Kasai, S. and Yamomoto, S. (1981) Establishment of a Clonal Osteogenic Cell Line from Newborn Mouse Calvaria. Japanese Journal of Oral Biology, 23, 899-901. http://dx.doi.org/10.2330/joralbiosci1965.23.899

[5] Sudo, H., Kodama, H.A., Amagai, Y., Yamamoto, S. and Kasai, S. (1983) In Vitro Differentiation and Calcification in a New Clonal Osteogenic Cell Line Derived from Newborn Mouse Calvaria. The Journal of Cell Biology, 96, 191-198. http://dx.doi.org/10.1083/jcb.96.1.191

[6] Chang, Y.L., Stanford, C.M. and Keller, J.C. (2000) Calcium and Phosphate Supplementation Promotes Bone Cell Mineralization: Implications for Hydroxyapatite (HA)-Enhanced Bone Formation. Journal of Biomedical Materials Research, 52, 270-278. http://dx.doi.org/10.1002/1097-4636(200011)52:2<270::AID-JBM5>3.0.CO;2-1

[7] Aaron, J.E. and Pautard, F.G.E. (1973) A Cell Cycle in Bone Mineralization. In: Balls, M. and Billett, F.S., Eds., The Cell Cycle in Development and Differentiation, University Press, Cambridge, 325-330.

[8] Aaron, J.E. and Pautard, F.G.E. (1975) Tetracycline Staining of Bone in Normal and Pathological States. In: Kuhlencordt, F. and Kruse, H.P., Eds., Calcium Metabolism, Bone and Metabolic Bone Diseases, Springer-Verlag, Heidelberg, Berlin, New York, 211-217. http://dx.doi.org/10.1007/978-3-642-80875-3_36

[9] Aaron, J.E., Makins, N.B. and Francis, R.M. (1984) Staining of the Calcification Front in Human Bone Using Contrasting Fluorochromes in Vitro. Journal of Histochemistry Cytochemistry, 32, 1251-1261. http://dx.doi.org/10.1177/32.12.6209330

[10] Perrin, D.D. (1965) Binding of Tetracyclines to Bone. Nature (London), 208, 787-789. http://dx.doi.org/10.1038/208787a0

[11] Kaitila, I., Wartiovaara, J., Laitinen, O. and Saxon, L. (1970) The Inhibitory Effect of Tetracycline on Osteogenesis in Organ Culture. Journal of Embryology and Experimental Morphology, 23, 185-211.

[12] Moremen, K.W. and Touster, O. (1986) Topology of Mannosidase II in Rat Liver Golgi Membranes and Release of the Catalytic Domain by Selective Proteolysis. The Journal of Biological Chemistry, 261, 10945-10951.

[13] Kashiwa, H. (1970) Calcium Phosphate in Osteogenic Cells. Clinical Orthopaedics, 70, 200-211.

[14] Oda, K., Hirose, S., Takami, N., Misumi, Y., Takatsuki, A. and Lkehara, Y. (1987) Brefeldin A Arrests the Intracellular Transport of a Precursor of Complement C3 Before its Conversion Site in Rat Hepatocytes. Federation of European Biochemical Societies, 214, 135-138. http://dx.doi.org/10.1016/0014-5793(87)80028-5

[15] Aaron, J.E. (1973) Osteocyte Types in the Developing Mouse Calvarium. Calcified Tissue Research, 12, 259-279. http://dx.doi.org/10.1007/BF02013740

[16] Aaron, J.E. (1974) The Development of the Bone Cell and its Role in Mineralization and Resorption. Ph.D. Dissertation, University of Leeds, Leeds.

[17] Fallon, V. (2006) The Fabrication of Mineral Particles by Bone Cells and Unicellular Organisms. Ph.D. Dissertation, University of Leeds, Leeds.

[18] Linton, K.M., Tapping, C.R., Adams, D.G., Carter, D.H., Shore, R.C. and Aaron, J.E. (2013) A Silicon Cell Cycle in a Bacterial Model of Calcium Phosphate Mineralogenesis. Micron, 44, 419-432. http://dx.doi.org/10.1016/j.micron.2012.09.008

[19] Davies, J.E., Chernecky, R., Lowenberg, B. and Shiga, A. (1991) Deposition and Resorption of Calcified Matrix in Vitro by Rat Marrow Cells. Cells Materials, 1, 3-15.

[20] Aaron, J.E. and Pautard, F.G.E. (1972) Ultrastructural Features of Phosphate in Developing Bone Cells. Israel Journal 
of Medical Sciences, 8, 625-629.

[21] Aaron, J.E., Oliver, B., Clarke, N. and Carter, D.H. (1999) Calcified Microspheres as Biological Entities and Their Isolation from Bone. The Histochemical Journal, 31, 445-470. http://dx.doi.org/10.1023/A:1003707909842

[22] Carter, D.H., Scully, A.J., Hatton, P.V., Davies, R.M. and Aaron, J.E. (2000) Cryopreservation and Image Enhancement of Juvenile and Adult Dentine Mineral. The Histochemical Journal, 32, 253-261. http://dx.doi.org/10.1023/A:1004059219242

[23] Wada, Y., Kataoka, H., Yokose, S., Ishizuya, T., Miyazono, K., Gao, Y.H., Shibasaki, Y. and Yamaguchi, A. (1998) Changes in Osteoblast Phenotype During Differentiation of Enzymatically Isolated Rat Calvaria Cells. Bone, 22, 479485. http://dx.doi.org/10.1016/S8756-3282(98)00039-8

[24] Carter, D.H., Scully, A.J. and Aaron, J.E. (1998) Evidence for Phosphoprotein Microspheres in Bone. The Histochemical Journal, 30, 677-686. http://dx.doi.org/10.1023/A:1003490506980

[25] Shahtaheri, M.S. and Aaron, J.E. (2001) Immunolocalization of Osteocalcin in Calcified Microspheres in Adult Mouse Bone Using FITC-Labelling. Bone, 28, 108S.

[26] Carter, D.H., Scully, A.J., Heaton, D.A., Young, M.P.J. and Aaron, J.E. (2002) Effect of Deproteination on Mineral Morphology: Implications for Biomaterials and Aging. Bone, 31, 389-395. http://dx.doi.org/10.1016/S8756-3282(02)00840-2

[27] Gerdes, H. and Kaether, C. (1996) Green Fluorescent Protein: Applications in Cell Biology. Federation of European Biochemical Societies, 389, 44-47.

[28] Tamura, G., Ando, K., Suzuki, S., Takatsuki, A. and Arima, K. (1968) Antiviral Activity of Brefeldin A and Verrucarin A. The Journal of Antibiotics, 21, 160-161. http://dx.doi.org/10.7164/antibiotics.21.160

[29] Klausner, R.D., Donaldson, J.G. and Lippincott-Schhwartz, J. (1992) Brefeldin A: Insights into the Control of Membrane Traffic and Organelle Structure. The Journal of Cell Biology, 116, 1071-1080. http://dx.doi.org/10.1083/jcb.116.5.1071

[30] Lu, Z., Joseph, D., Bugnard, E., Zaal, K.J.M. and Ralston, E. (2001) Golgi Complex Reorganization During Muscle Differentiation: Visualization in Living Cells and Mechanism. Molecular Biology of the Cell, 12, 795-808. http://dx.doi.org/10.1091/mbc.12.4.795

[31] Pautard, F.G.E. (1961) Calcium Phosphate and the Origin of Backbones. New Scientist, 12, 364-366.

[32] Fallon, V., Garner, P.E. and Aaron J.E. (2014) Stress-Induced Golgi Activity and Mineralogenesis: Spirostomum ambiguum as a Protozoan Model for Bone? Submitted. 
Scientific Research Publishing (SCIRP) is one of the largest Open Access journal publishers. It is currently publishing more than 200 open access, online, peer-reviewed journals covering a wide range of academic disciplines. SCIRP serves the worldwide academic communities and contributes to the progress and application of science with its publication.

Other selected journals from SCIRP are listed as below. Submit your manuscript to us via either submit@scirp.org or Online Submission Portal.
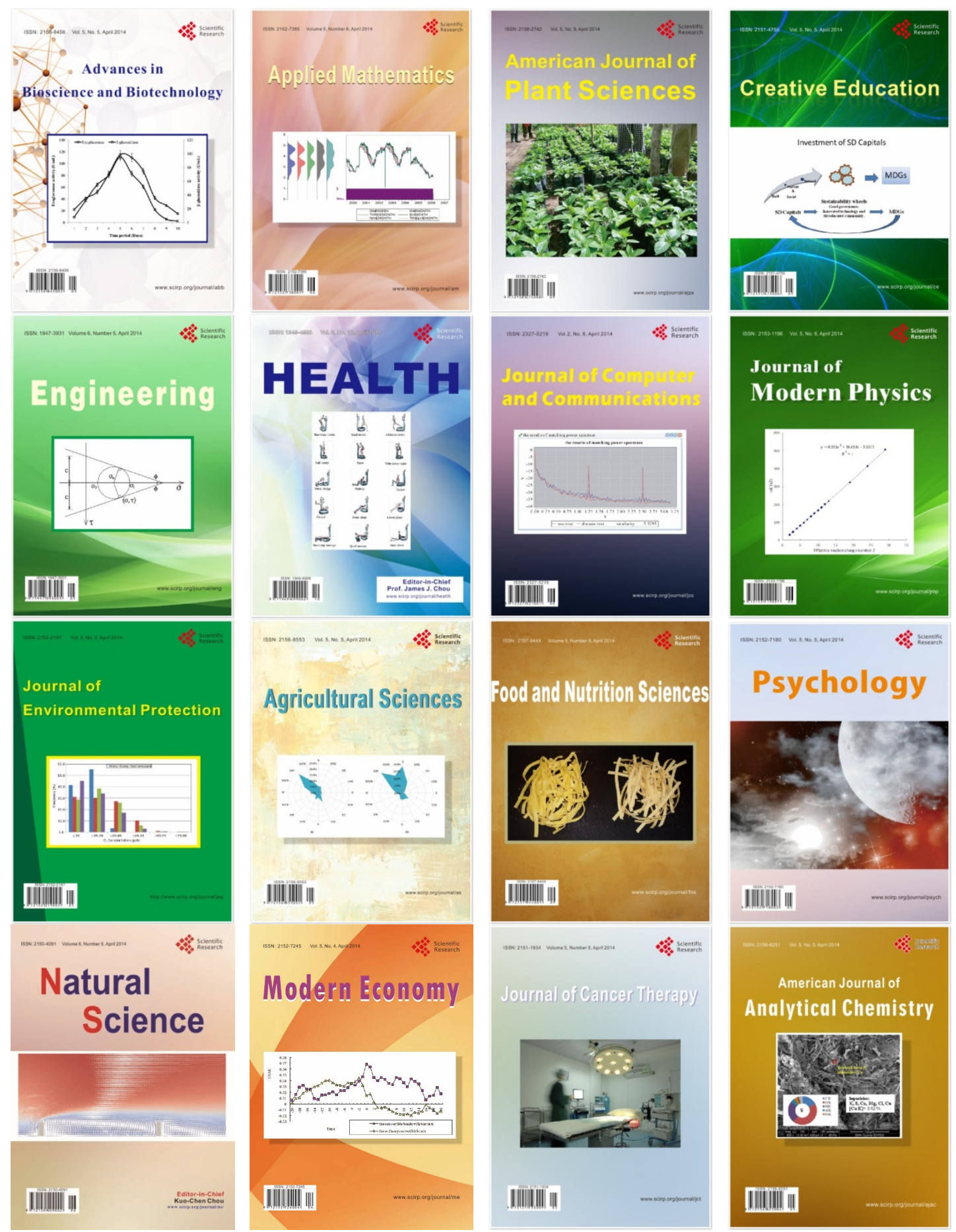